\title{
Prevalence and clinical significance of pancreatic cystic lesions in immunosuppressed patients with solid organ transplantation
}

\author{
Young-Dong Yu' ${ }^{1}$, Pyoung-Jae Park ${ }^{2}$, Hye-Sung Jo ${ }^{1}$, Dong-Sik Kim² \\ ${ }^{1}$ Division of Hepatobiliary, Department of Surgery, Korea University Anam Hospital, Seoul, Korea \\ ${ }^{2}$ Division of Transplantation, Department of Surgery, Korea University Guro Hospital, Seoul, Korea
}

Background: Solid organ transplant recipients have an increased risk of cancer due to immunosuppressive therapy. Pancreatic cystic lesions (PCLs) are increasingly being detected, some with malignant potential. We aimed to determine the prevalence of these lesions and describe their clinical course in these patients.

Methods: We identified the presence of PCLs in a retrospective cohort of 804 consecutive solid organ transplant recipients from 2009 to 2019 and compared lesion characteristics at initial and follow-up imaging, when available. We also compared these features with an immunocompetent control group encompassing patients under surveillance for greater than 12 months and were matched for age and sex.

Results: There were 15 patients in the study group and 60 patients in the control group. Among the solid organ transplant recipients with PCLs, there were seven and eight patients undergoing liver and kidney transplantation, respectively. Lesion prevalence was $1.86 \%$ (15/805). Median diameter of the largest lesion was $20 \mathrm{~mm}$ (range, 0.2-60 mm) and most lesions were benign $(9 / 15$, simple cyst or pseudocyst). During follow-up imaging, the cysts size remained stable in $79.7 \%$, increased in $6.6 \%$, and decreased in $13.7 \%$. Among patients diagnosed with intraductal papillary mucinous neoplasm (6/15), worrisome features were noted in one patient at the time of cyst diagnosis. However, due to multiple comorbidities the patient received only conservative management. There were no significantly different features including the rate of size increase or the development of worrisome features between the study and control group $(P<0.05)$.

Conclusions: PCLs are somewhat common in solid organ transplant recipients. In lesions without high-risk features, the development of features worrisome for cancer is rare. These lesions can be managed conservatively, and their presence should not affect transplant eligibility. 